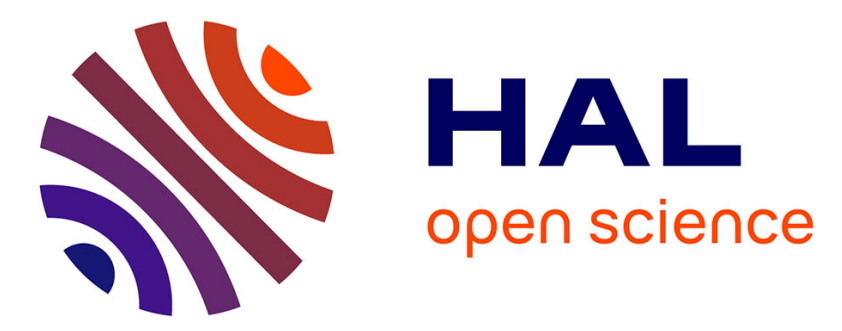

\title{
Formation of the radial electric field profile in the WEST tokamak
}

\author{
L. Vermare, P. Hennequin, C. Honoré, M. Peret, Guilhem Dif-Pradalier, X. \\ Garbet, J. Gunn, C. Bourdelle, F. Clairet, R. Varennes, et al.
}

\section{- To cite this version:}

L. Vermare, P. Hennequin, C. Honoré, M. Peret, Guilhem Dif-Pradalier, et al.. Formation of the radial electric field profile in the WEST tokamak. Nuclear Fusion, 2022, 62 (2), pp.026002. 10.1088/17414326/ac3c85 . hal-03441556

\section{HAL Id: hal-03441556 https://hal.science/hal-03441556}

Submitted on 22 Nov 2021

HAL is a multi-disciplinary open access archive for the deposit and dissemination of scientific research documents, whether they are published or not. The documents may come from teaching and research institutions in France or abroad, or from public or private research centers.
L'archive ouverte pluridisciplinaire HAL, est destinée au dépôt et à la diffusion de documents scientifiques de niveau recherche, publiés ou non, émanant des établissements d'enseignement et de recherche français ou étrangers, des laboratoires publics ou privés. 


\section{- Formation of the radial electric field profile in the 2 WEST tokamak}

L. Vermare, P. Hennequin, C. Honoré

Ecole Polytechnique, LPP, CNRS UMR 7648,91128 Palaiseau, France

M. Peret, G. Dif-Pradalier, X. Garbet, J. Gunn, C. Bourdelle, F. Clairet, R. Varennes, J. Morales, R. Dumont, M. Goniche, P. Maget and WEST Team http://west.cea.fr/westteam CEA, IRFM, F-13108 Saint-Paul-Lez-Durance, France 


\section{Abstract}

Sheared flows are known to reduce turbulent transport by decreasing the correlation 10 length and/or intensity of turbulent structures. The transport barrier that takes place at the edge during improved regimes such as $\mathrm{H}$ mode, corresponds to the establishment of a large shear of the radial electric field. In this context, the radial shape of the radial electric field or more exactly of the perpendicular $E \times B$ velocity appears as a key element in accessing improved confinement regimes. In this paper, we present the radial profile of the perpendicular velocity measured using Doppler back-scattering system at the edge of the plasma, dominated by the $E \times B$ velocity, during the first campaigns of the WEST tokamak. It is found that the radial velocity profile is clearly more sheared in Lower Single Null configuration (with the $B \times \nabla B$ magnetic drift pointing toward the active X-point) than in Upper Single Null configuration for ohmic and low current plasmas $\left(B=3.7 T\right.$ and $\left.q_{95}=4.7\right)$, consistently with the expectation comparing respectively "favourable" versus "unfavourable" configuration. Interestingly, this tendency is sensitive to the plasma current and to the amount of additional heating power leading to plasma conditions in which the $E \times B$ velocity exhibits a deeper well in USN configuration. For example, while the velocity profile exhibits a clear and deep well just inside the separatrix concomitant with the formation of a density pedestal during L-H transitions observed in LSN configuration, deeper $E_{r}$ wells are observed in USN configuration during similar transitions with less pronounced density pedestal.

\section{Introduction}

The shear of the radial electric field at the edge is widely accepted to be responsible 30 for turbulence reduction in edge transport barriers [Bur+89] and thought as a key ingredient of the improved confinement of $\mathrm{H}$-mode plasmas [Wag+82]. Experimental evidence that shear flow plays a crucial role in triggering and maintaining edge transport barrier in H-mode regime has been largely demonstrated (for example in DIII-D [BDT90], ALCATOR C-MOD [McD+09], TJ-II [Est+09], ASDEX Upgrade [Vie+13], JET [And+08], JT60 [Kam+11], and for a review see [Bur20]. The radial electric field measured just inside the separatrix is found to be dominated by the diamagnetic contribution in ALCATOR C-MOD H mode [McD+09] as well as in ASDEX Upgrade both in H-mode [Vie+13] and during I-phase $[\mathrm{Cav}+16]$. In the other hand, approaching the L-H transition, contributions related to turbulence generated flows are reported to enter at play $[\mathrm{Con}+11 ; \mathrm{Sch}+12$; Est +11$]$.

Nevertheless, a full understanding of how the $E_{r}$ profile builds up at the edge is still lacking. It can be formulated either as the result of a competition between several mechanisms that generate and damp flows or as the result of a non-ambipolar particle flux, which enhances radial charge separation imposing a radial electric field profile. In neoclassical theory, in an axisymmetric plasma, the thermal radial fluxes are intrinsically ambipolar. As a result, the radial electric field $E_{r}$ is undetermined. 
The toroidal $v_{\varphi}$ and the poloidal $v_{\theta}$ velocities can be determined by projecting the momentum balance equations in the toroidal (or equivalently parallel) and poloidal directions, admitting that the radial force balance equation is satisfied on a short time scale. Standard neoclassical theory predicts the parallel component of a collisional stress tensor. The parallel force balance equation then leads to a prediction on the poloidal velocity proportional to the main ion temperature gradient- more precisely $v_{\theta}=$ $-\frac{K}{m_{i} \Omega_{i}} \frac{d T_{i}}{d r}$ [KDG91], valid when impurities are trace. However the toroidal component of the neoclassical stress tensor is vanishingly small. As a consequence, ambipolarity is automatically satisfied and the toroidal velocity cannot be determined separately from the radial electric field. Therefore, in this context (no external momentum source, axisymmetric plasma), the neoclassical theory does not predict an independent toroidal rotation. The contribution from the perpendicular velocity $v_{s, \perp}=v_{s, \theta} \frac{B_{\varphi}}{B}-v_{s, \varphi} \frac{B_{\theta}}{B}$ is then not expected to be negligible.

At this point, two equations remain: the time evolution of the radial electric field, which can be written as

$$
\epsilon \frac{\partial E_{r}}{\partial t}=-j_{r}
$$

where $\epsilon$ is the dielectric constant in toroidal plasmas and $j_{r}$ the total radial current which includes several contributions such as the neoclassical viscosity current related to collisions, the orbit loss current and the current related to the turbulent Reynolds stress.

In addition, the radial force balance couples the ion toroidal velocity and the radial electric field at given poloidal velocity and pressure gradient and reads :

$$
E_{r}=v_{s, \varphi} B_{\theta}-v_{s, \theta} B_{\varphi}+\frac{\nabla p_{s}}{n_{s} q_{s}}
$$

Therefore, from here one can see this equation as an expression of the toroidal velocity as a function of the radial electric field and then use the equation 1 to determine the radial electric field. Another way to proceed, and the one chosen in this paper, is to see the radial electric field as the sum of three contributions : one coming from the toroidal rotation, one from the poloidal rotation and the diamagnetic contribution.

In some specific cases, the diamagnetic contribution is found to be dominant (as mentioned above [Vie+13; Cav+16]. This is the the so-called "neoclassical prediction" , which is also obtained when assuming a strong neutral firction force, as mentioned for example in [SMR11]. In this regime, the neutral friction force $F_{n j}=-n m_{j}^{1 / 2} \nu_{n j} u_{\perp j}$ that creates a radial drift which combined with the ambipolary leads to a $E_{r}$ such that $F_{n i}=-F_{n e}$. This balance leads to an expression for $E_{r}$ that depends on $\nu_{n i} / \nu_{n e}$, $\nabla P_{e} / \nabla P_{i}$ and the mass ratio $\epsilon=m_{e} / m_{i}$. The case of $T_{e} \approx T_{i}, \nu_{n i} \approx \nu_{n e}$ leads to a zero perpendicular velocity. As a result, the radial electric field can be reduced to the diamagnetic radial electric field : 


$$
E_{r, d i a}=\frac{\nabla p_{i}}{n_{i} q_{i}}
$$

However, it should be emphasized that this approximation/prediction is based 83 on strong assumptions and by consequence its validity domain holds as long as the ${ }_{84}$ friction force dominates. A full self-consistent prediction of plasma rotation requires an 85 independent theoretical prediction for $E_{r}$, which is typically the result of momentum 86 transport on time scales longer that the ion collisional time. Among possible mechanisms ${ }_{87}$ to be taken into account, one can think in terms of neoclassical effects from one side, 88 such as ion orbit losses [CKW02; dMB11; Brz+19], toroidal magnetic ripple [Gar+10; 89 Nav+10; Ura+11; Fen+11] and as already mentioned the effect of neutral friction 90 $[$ Mon+97; SMR11] at the edge or, on the other side, turbulent generation of poloidal 91 momentum [DK91; Dif+09; Gri+13].

In addition, magnetic drift toward the X-point is commonly considered as the ${ }_{93}$ favourable configuration to access an H-mode regime with the lowest power threshold. 94 While there are multiple proposed explanations $[\mathrm{Fed}+12$; Cha+17], there is no clear 95 consensus on the reason of such favourable versus unfavourable configuration and 96 systematic experimental comparisons of velocity profiles in USN and LSN are scarce. $\quad 97$

The present contribution gives a first review of radial electric field profiles at 98 the edge of the WEST tokamak in both LSN and USN configuarions during its first 99 campaigns that may bring some complementary elements helping in the understanding 100 of $E_{r}$ formation, while approaching the L-H transition. 101

The WEST tokamak is an upgrade of Tore Supra from a limiter-based tokamak 102 with carbon PFCs into an X-point divertor tokamak with full-tungsten armour while ${ }_{103}$ keeping its long pulse capability [Buc +14$]$. As a result, WEST is a large aspect ratio ${ }_{104}$ machine $(\mathrm{A}=5-6)$ with a magnetic ripple around $3 \%$ at the plasma outboard edge, lower ${ }_{105}$ than in Tore Supra (around 6\%). Additional heating is based on RF systems (both 106 Lower Hybrid and Ion Cyclotron Resonance Heating systems); divertors are symmetric ${ }_{107}$ with active $\mathrm{X}$-point either at the bottom or at the top and the $B \times \nabla B$ drift always ${ }_{108}$ pointing down (as visible in Figure 1). 109

In order to place the context, previous results obtained on Tore Supra show that ${ }_{110}$ the radial profile of the perpendicular flow could be separated in three spatial areas. ${ }_{111}$ Inside $r / a=0.8$, the radial electric field is dominated by losses of thermal ions due to ${ }_{112}$ the magnetic ripple [Tri+08] while between $0.7<r / a<0.95$, a competition between ${ }_{113}$ the latter and the generation of large scale flows by turbulence appears as a possible ${ }_{114}$ explanation of the measured poloidal asymmetry of the mean perpendicular velocity 115 [Ver+18]. In addition, edge conditions such as contact points and parallel dynamics in 116 the scrape-off-layer (SOL) influence the edge profiles beyond $r / a=0.9[$ Hen +10$] . \quad{ }_{117}$

The velocity profiles presented and discussed in the following are obtained from 118 Doppler BackScattering (DBS also called Doppler reflectometry) measurements in X- ${ }_{119}$ mode polarisation $[$ Hen +06$]$. This system probes the plasma using microwaves launched 120 in oblique incidence and stepped in frequency. The signal collected by the antenna ${ }_{121}$ 
corresponds mainly to the signal backscattered by density fluctuations in the vicinity of the cut-off layer. The detected density fluctuations are selected at a spatial scale given by the incidence angle (and by the propagation in the plasma toward the cut-off layer). In the context of velocity measurements at the edge, density fluctuations act as tracers to access the $E \times B$ velocity. The phase velocity that corresponds to the velocity of fluctuations in the plasma frame is found negligible in the area of interest here (for $\left.\rho_{p o l}>0.9\right)$ by performing probing wavenumber scans. In the standard measurements setup, each radial point of the profile corresponds typically to an averaged velocity over $7 \mathrm{~ms}$ leading to an entire profile (i.e 12 probing frequency steps) evaluated on approximately $80 \mathrm{~ms}$. For the reconstruction of velocity profiles, the measurements location and the wavenumber selected at the turning point are computed using a beam tracing code $[$ Hon +06$]$; the input density profile is measured using using fast sweep reflectometry in $\mathrm{X}$-mode polarization $[\mathrm{Cla}+17]$ in burst mode (ie. each probing frequency ramp is swept in $1 \mu s$ and each burst contains around 8000 profiles) and it corresponds to an average over 200 profiles regularly distributed in a full burst (i.e average over 10ms) with an input equilibrium contrained using the equilibrium reconstruction code NICE (Newton direct and Inverse Computation for Equilibrium) [Fau20].

The paper is organized as follows. The first section is devoted to the comparison of the radial profile of the perpendicular velocity in Lower Single Null (LSN) configuration versus Upper single Null (USN) configuration in low power plasmas. Then, the evolution of these velocity profiles during L-H transition in both, LSN and USN, configurations are presented respectively in section 3 and 4 . These first observations in WEST plasmas are finally discussed in the last section.

\section{Velocity profile in LSN and USN configurations during ohmic discharges}

This section is dedicated to ohmic discharges which allows investigations on the radial electric field profiles with long stationnary phases and scanning properly several plasma parameters (magnetic configuration, plasma current, density...). The comparison between low and high power discharges in different magnetic configurations brings elements to understand the balance and the weigth of each mechanisms at work in the formation of the $E_{r}$ profile. One can argue that the plasma conditions in ohmic phases are far from those of L-H transition but it should be noted that the set of possible mechanisms that come into play for generating $E_{r}$ are not expected to change between both regimes, only their respective weights are.

In WEST plasmas, with no heating power (also valid at low power), the radial profile of the $E \times B$ velocity between LSN and USN plasmas shows an impressive difference (see Fig 1 (left)). In LSN configuration, the $E \times B$ velocity profile exhibits a "standard shape" : a positive velocity outside the separatrix (i.e. in the ion diamagnetic direction), changing sign across the separatrix and forming a well in the edge of the confined plasma with a velocity that decreases in absolute value toward the core region. In contrast, the radial profile for USN configuration does not show a well and exhibits 
a smooth decay from the edge to the core. Interestingly, the density and electron 162 temperature $T_{e}$ profiles (no ion temperature $T_{i}$ measurement are available at this stage) ${ }_{163}$ for these discharges, which are presented in Figure 1 (right), are very similar at the ${ }_{164}$ edge. The energy content, $W_{M H D}$ is also identical for both discharges. However, it ${ }_{165}$ should be mentionned that the safety factor $q$ and the internal inductance are slighly ${ }_{166}$ different ; in the LSN configuration, $q$ at the edge (as inferred from magnetic equilibrium 167 reconstruction constrained by polarimetry) is higher by about $20 \%$ while $l_{i}$ is lower by ${ }_{168}$ about $30 \%$.

Approximating the diamagnetic radial electric field (i. e. $E_{r} \approx \nabla P_{i} / e n_{i},{ }_{170}$ eq. 3) using the electron pressure, it appears that this contribution is not the ${ }_{171}$ dominant contribution, as least in the USN configuration. Especially, the shape of 172 the density gradient exhibits a well suggesting that the velocity profile deviate from this ${ }_{173}$ approximation.
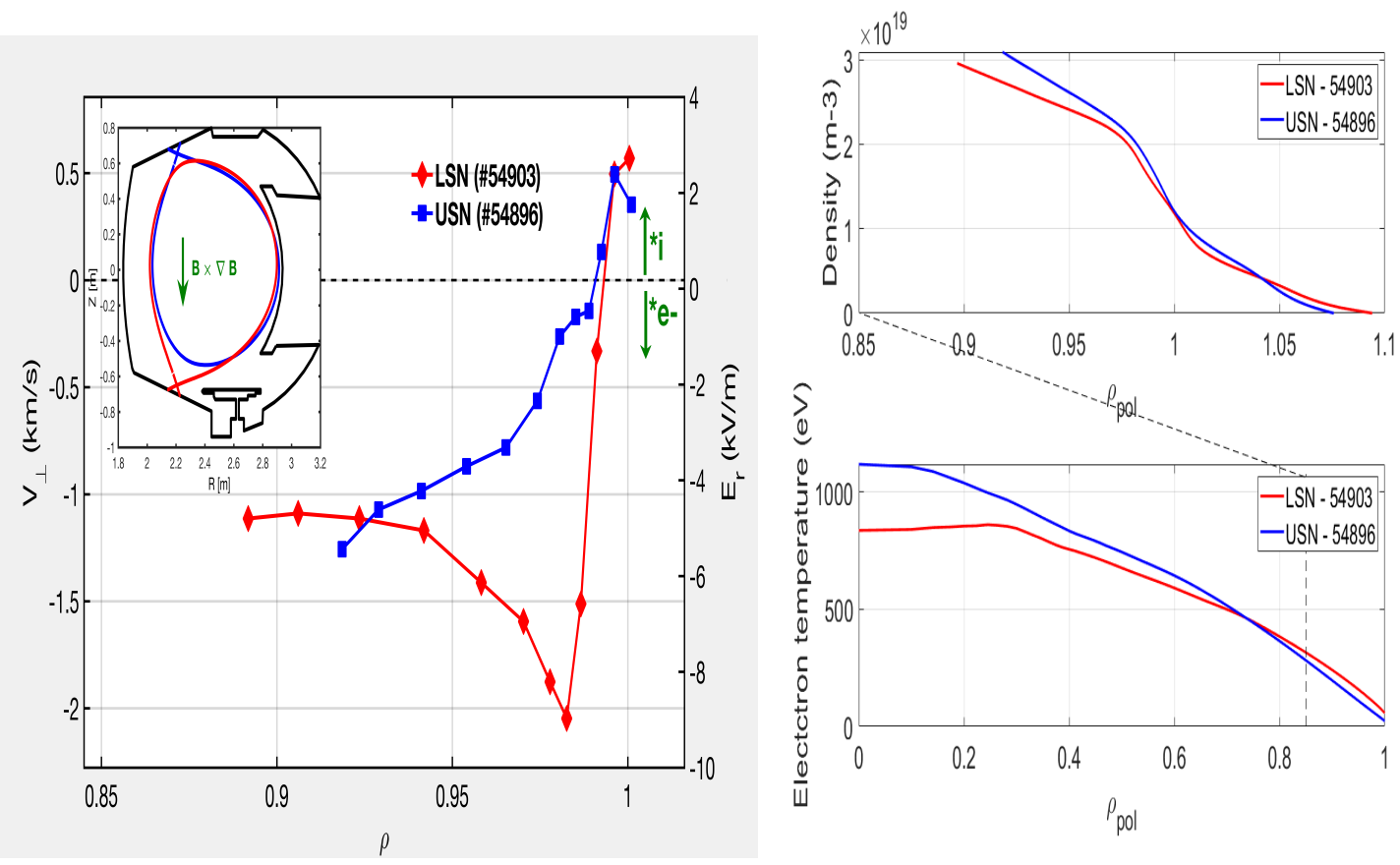

Figure 1. Radial profiles of perpendicular velocity and corresponding $E_{r}$ profiles measured using DBS in LSN (red) and in USN (blue) configurations during ohmic plasmas (left). Radial density profiles measured using fast sweep reflectometry $[\mathrm{Cla}+17]$ and electron temperature mesaured using ECE radiometer during the same discharges (right).

Concerning the difference in the shape of the velocity profile, a similar observation 175 has been obtained in pure ICRH heated ASDEX Upgrade plasmas [Sch+06] and ${ }_{176}$ interestingly also in Tore Supra circular plasmas during low current ohmic discharges 177 $[$ Fed $+13 ;$ Hen +10$]$. In the latter, the contact point with this limiter is rolled from 178 $\theta_{X} \approx 35^{\circ}$ below the midplane (equivalent to LSN) to $\theta_{X} \approx+35^{\circ}$ above the midplane ${ }_{179}$ (equivalent to USN). The modification of the velocity shape has been shown to be ${ }_{180}$ 
compatible with a simplified model based on the dynamical interaction between potential eddies and shear flow $[\mathrm{Fed}+13]$. When eddies are tilted in the velocity shear direction, the system is favourable to shear increase while when they are tilted in the opposite direction, the shear flow is damped. Therefore, depending of the position of the contact point, the balance between both contributions (associated with the change of the tilting direction between upper and lower section of the low field side part of the plasma) is modified and the averaged velocity profile is modified. It is important to notice that this mechanism depends on the magnetic shear.
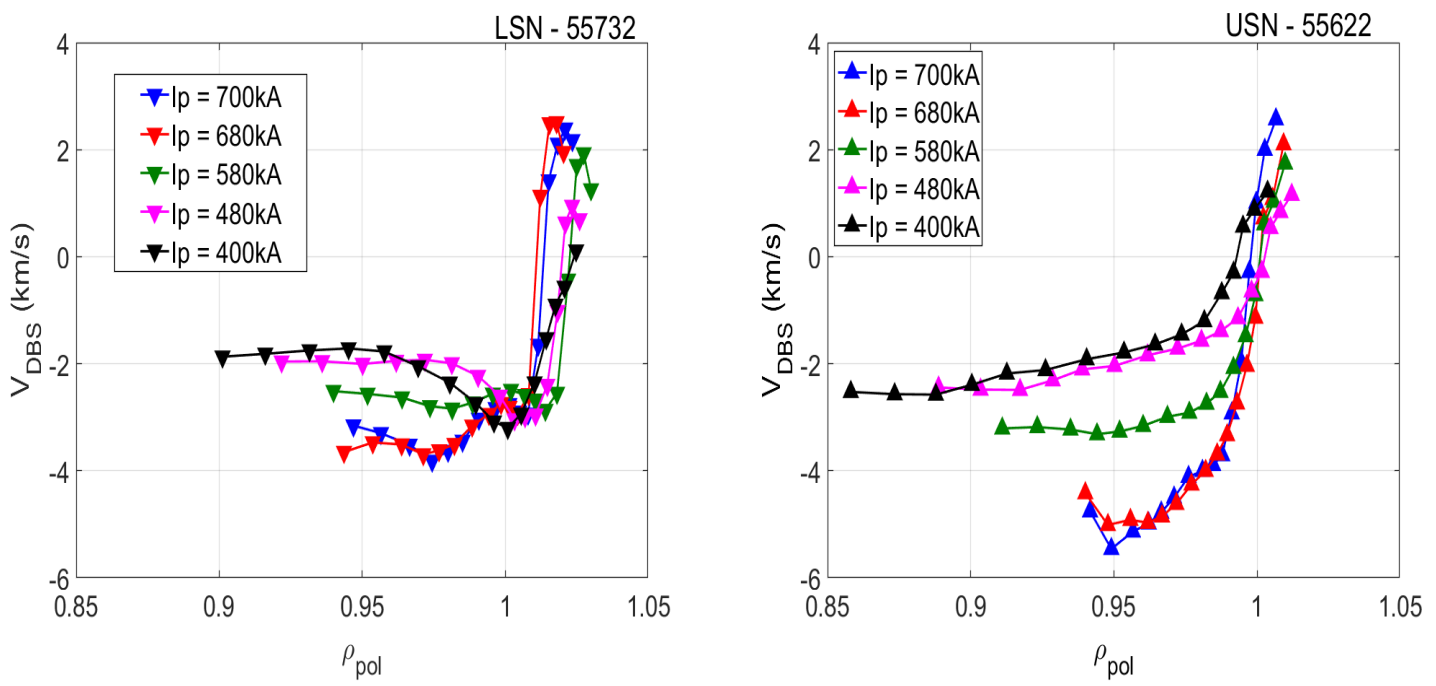

Figure 2. Radial profiles of perpendicular velocity $V_{E \times B}$ measured using DBS varying the plasma current $I_{p}$ in LSN (left) and in USN (right) configurations during ohmic plasmas.

Even if plasma conditions are far from L-H transition, the difference observed in the velocity shear between LSN versus USN may appear consistent with the fact that the configuration with the $B \times \nabla B$ magnetic drift pointing toward the active $\mathrm{X}$-point, designated as the 'favourable configuration', is more favourable to reach the H-mode as compared to the opposite case when $B \times \nabla B$ magnetic drift points away from the active $\mathrm{X}$-point.

However, this tendency is sensitive to the plasma parameters. In particular, experiments have been performed to compare the DBS velocity profiles at the edge in both USN and LSN at different plasma current $I_{p}$ keeping $B=3.7 T$. During this scan the safety factor is varying by a factor 1.7 with a small difference between USN and LSN discharges in the safety factor covered range such as $q_{95}=[3.3-5.8]$ in LSN while $q_{95}=[2.85-4.7]$ in USN. Note that during these scans, it is found from the radial profiles of the safety factor that the magnetic shear does not change significantly. As visible in Figure 2, it appears that the plasma current impacts significantly the velocity profile in the USN discharges. When increasing the plasma current, the $E \times B$ velocity starts to form a well to end up with a deeper profile than in LSN at high current. Indeed, 
in LSN configuration, the increase of the plasma current also deepens the radial electric ${ }_{205}$ field well ; however, the effect is less pronounced than in USN. While the density profile 206 also slightly changes during the $I_{p}$ scan, it is found that the dominant effect comes ${ }_{207}$ from the plasma current variation. These results suggest that, at least one dominant 208 mechanism entering in the $E_{r}$ profile formation in USN depends on the plasma current ${ }_{209}$ or the safety factor.

Interestingly, for fixed plasma current and moderate to high heating power, the ${ }_{211}$ velocity profile ends up deeper in the USN configuration for the same plasma conditions. 212 This result leads to an unexpected situation in which the "unfavourable configuration" ${ }_{213}$ seems more favourable, at least from the point of view of mechanisms for improved 214 confinement based on vortex shearing. This situation is addressed in the next session, 215 in the context of L-H transitions.

\section{Evolution of the radial electric field profile during L-H transition}

\subsection{L-H transition observed in LSN configuration}

In WEST, up to now, L-H transitions have been observed after fresh boronization (when ${ }_{219}$ the radiated fraction inside the separatrix is lower than 40\%) with heat power crosses 220 the separatrix $P_{\text {sep }}$ close to the ITPA scaling law prediction [MTI08], as visible in the ${ }^{221}$ Figure 3. While no sign of an established H-mode regime is visible (such as ELMs), 222 transitions have been identified by simultaneously observing expected changes in both ${ }_{223}$ the confined and SOL plasmas. Time traces of this discharge in LSN configuration (i.e. ${ }^{224}$ with 'favourable $B \times \nabla B$ ' directed towards the lower divertor) with an L-H transition ${ }_{225}^{22}$ occurring at $t=6.45 \mathrm{~s}$ are shown in Figure 4 . This is a discharge using both LHCD and 226 ICRH additional power $P_{\text {sep }}=3.8 \mathrm{MW}$ with $B_{T}=3.7 T, I_{p}=0.5 \mathrm{MA}$ and $q_{95}=4.5$. ${ }^{227}$ In the confined plasma, edge steepening of the density profile (from both reflectometry ${ }_{228}$ and interferometry measurements), increase of the total energy content $W_{M H D}$, decrease ${ }_{229}$ internal inductance (as expected in case of increased edge bootstrap current) and a 230 deepening of the $E_{r}$ well measured by Doppler Back Scattering are simultaneously ${ }_{231}$ observed.

In the SOL, concurrently to the changes mentioned above in the confined plasma, 233 several modifications are observed. A reduced transport flux was measured such as ${ }^{234}$ reduced D-gamma signal on the divertor target. In the same time, the density measured ${ }^{235}$ by the SOL interferometer chord exhibits a reduction as well as the flux measured on the ${ }_{236}$ Langmuir probes at the divertor targets (measurements available in LSN only for this ${ }^{237}$ experiment) and the gas puff rate. Note for now that no measurements of the pedestal 238 electron temperature are available since the ECE signal is polluted by the fast electrons ${ }_{239}$ from LHCD for $\rho_{\text {pol }}>0.5$. In the four LSN discharges presenting an L-H transition, the 240 power crossing the separatrix remains close to the threshold leading to a more or less ${ }_{241}$ pronounced oscillatory phase. In the present work, we focused on one LSN discharge ${ }_{242}$ during which fast reflectometry measurements are available to access high-resolution ${ }^{243}$ 


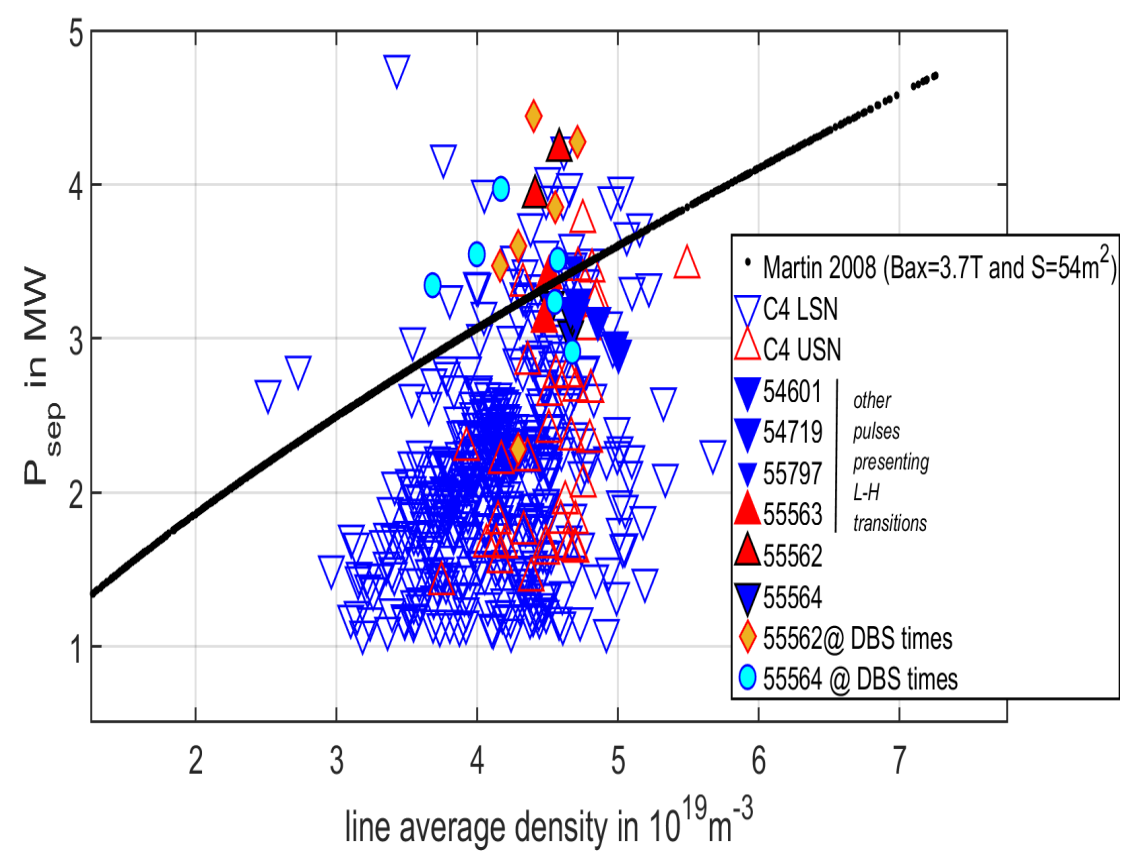

Figure 3. Heat power crossing the separatrix as a function of the line average density of WEST campaign $\mathrm{C} 4$ discharges for $I_{p}=0.5 M A$ and $B=3.7 T$. Time sequences in LSN configuration (red) and in USN configuration (blue), symbols with black line corresponding to the discharges, respectively 55564 and 55562, and full symbolds corersponding to other L-H transition discharges

density profiles and with moderate density at the edge in order to probe a sufficient wide edge area. During this discharge, the increment in the total energy content $W_{M H D}$ is about $20 \%$ between time phase just before and the one just after the transition. The confinement time normalized to the ITER scaling law (which gives the confinement time $\tau_{\text {ITER-98(y,2) }}$ [CCE99]), known as the $\mathrm{H}$ factor labelled as $H_{98}$ is around 0.6. This rather low value is explained by the high level of radiated power. Computing this $\mathrm{H}$-factor subtracting the radiated power to the total power gives $H_{98, P_{t o t}-P_{\text {rad }}}=0.8$. Note that the transitions presented in this paper are probably in the low-density branch [Ryt+13].

As mentioned above and visible in Figure 4 , the transition leads to an oscillatory phase that can be explained by the fact that, at the transition at $t=6.45 \mathrm{~s}$, the density and the power radiated (frad) rises leading to a reduction of the power crossing the separatrix and then to a reduction of the density. Then while the density is reduced, the radiated power reduces as well, allowing for the power crossing the separatrix to rise again and for the pedestal to form again, etc. Similar oscillatory H-L transitions are reported in Deuterium plasmas in JET-ILW on the low density branch where the radiated freaction power is larger (40\%) [Vin19] as well as on AUG when operating well after a boronization [Put20].

Figure 5 shows that, from the transition time, the density profile exhibits a pedestal at the edge. At the same time, a clear deepening of the radial profile of the $E \times B$ velocity 

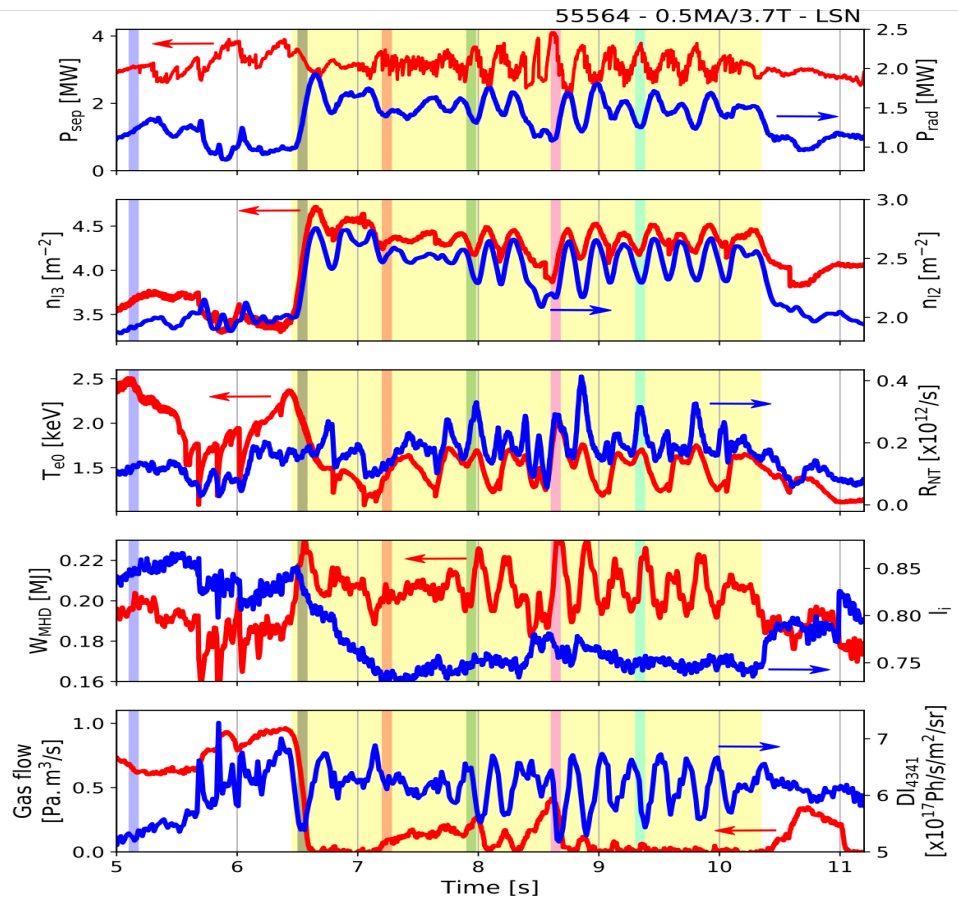

Figure 4. Time traces of radiated power $P_{\text {rad }}$ and power crossing the separatrix $P_{\text {sep }}$, central line integrated densities $n_{l 2}$ and $n_{l 3}$, central electron temperature and neutrons rate $R_{N T}$, total energy content $W_{M H D}$ and internal inductance $l_{i}$, gas flow rate and Dgamma signal on the divertor target $D_{I 4341}$ for a LSN discharge with an L-H transition at $t=6.45 \mathrm{~s}$. The yellow area shows the $\mathrm{L}-\mathrm{H}$ transition phase and colored vertical lines show the times of the DBS measurements

is visible. The radial electric field well remains deep during the oscillatory phase, at ${ }^{263}$ least at the time of DBS measurements. The radial profiles of the velocity are measured ${ }^{264}$ before $(t=5.1 s)$ and just after the transition $(t=6.5 s)$ and during the oscillatory phase ${ }_{265}$ $(t=7.2 s, 7.9 s, 8.6 s$ and $9.3 s)$.

Before discussing the specifics of the radial profiles presented in Figure 5, it 267 is necessary to mention that the sensitivity of the initialization of density profiles ${ }^{268}$ to pollution by fast electrons radiation (due to LHCD heating which generates 269 suprathermal electrons which create a broadband ECE stray radiation affecting the 270 signal to noise ratio of the fast sweep reflectometer $[\mathrm{Cla}+01])$ and the equilibrium 271 uncertainties (that can vary between LSN and USN configurations), can lead to a radial ${ }_{272}$ shift of the density profiles, varying from one sweep to another. In order to compensate ${ }^{273}$ this shift, averaged density profiles were slightly shifted. Choice has been made to ${ }^{274}$ align the profiles in order to get the main inflection point close to the separatrix for all ${ }_{275}$ profiles. While this hypothesis is far from satisfactory, it helps in making both figures ${ }_{276}$ (density and velocity profiles) more readable. However, it is important to notice that ${ }_{277}$ the radial shift applied modifies the reconstruction of the velocity profiles from DBS ${ }_{278}$ since the determination of the radial position and the probing wavenumber selected at 279 the turning point are computed using these profiles in a beam tracing code [Hon+06]. ${ }_{280}$ 

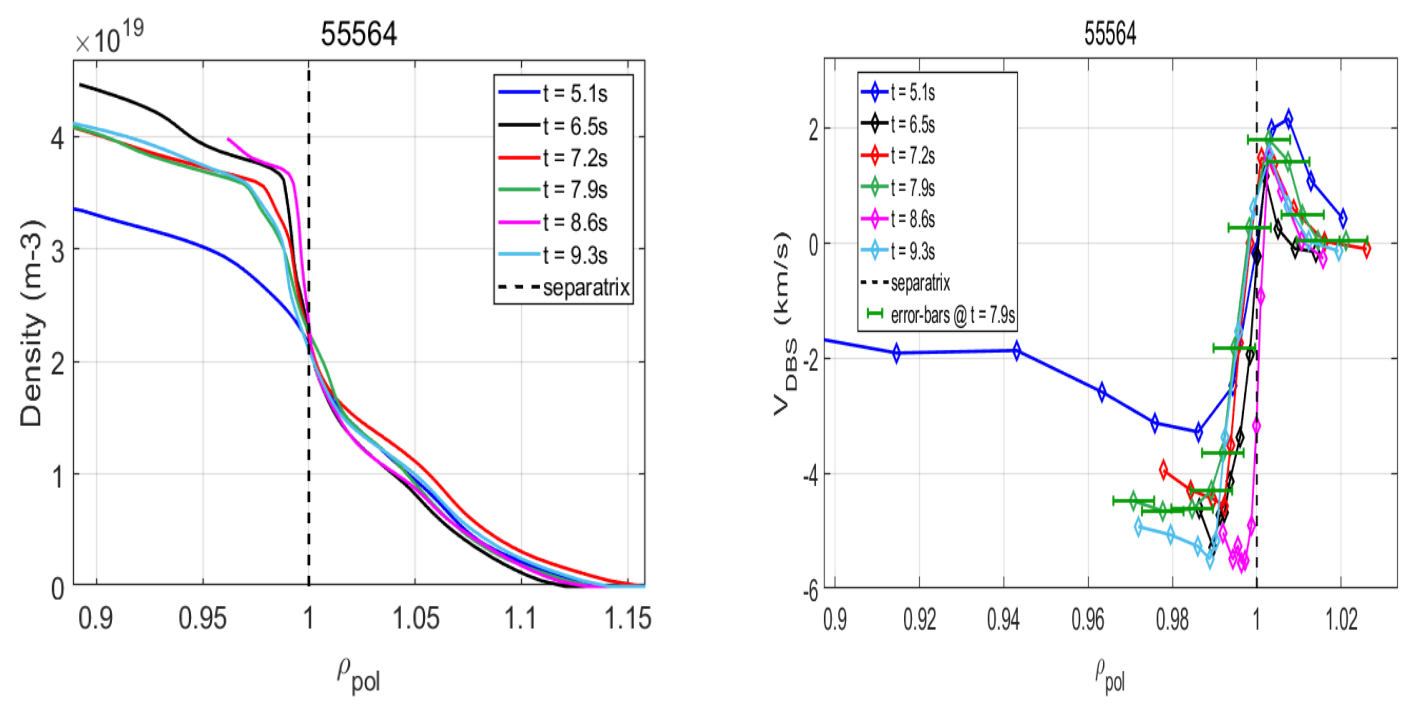

Figure 5. Radial profiles of electron density measured using fast sweep reflectometry $[\mathrm{Cla}+17]$ during an L-H transition phase in LSN starting at $6.45 \mathrm{~s}$ (left). Associated radial profiles of the $\mathrm{E} \times \mathrm{B}$ velocity measured using DBS [Hen+06] (right)

However, the radial shift applied to the density profile has a negligible impact on the value in term of velocity (i.e the amplitude of the velocity is not sensitive the shift of the density profile).

Coming back to the evolution of density profiles, it can be seen that a pedestal is formed from $t=6.5 \mathrm{~s}$ for which $P_{\text {sep }}=3.3 \mathrm{MW}$; it remains visible on each DBS time measurements during the whole sequence, even if it evolves slightly with small changes in additional heating power. Notably, the gradient weakly decreases at $t=7.2 \mathrm{~s}$, and $t=7.9 \mathrm{~s}$ while it is recovered at $t=8.6 \mathrm{~s}$ to finally decreases slightly again at $t=9.3 . \mathrm{s}$. Note that from $t=7.9 \mathrm{~s}$ density starts to strongly oscillate. While this behaviour is visible in the time traces showed in Figure 4, it is not on density profiles (averaged on $10 \mathrm{~ms}$ ) since the time of the DBS measurements does not resolve a full cycle of the oscillation. However, due to the time resolution of the DBS system (one entire profile requires approximatively $80 \mathrm{~ms}$ ), the density evolves during the velocity measurements. In order to evaluate the error-bars on the radial position (influence of the density profile in the velocity values through the wavenumber is negligible) of DBS measurements due to these oscillations, beam tracing has been performed for extreme density profile (at the extrema of the closet density oscillation) for one DBS time acquisition (see Figure 5).

On the velocity profiles, concomitantly to the formation of the density pedestal and as expected considering contribution $\nabla P$, a deeper and narrower well is formed as compared to the profile before the transition. While the well remains deep for all the measurements time (in several locations), the depth of the well and the shape change softly for the three times during which the density gradient slightly decreases. On the contrary, at $t=8.6 \mathrm{~s}\left(P_{\text {sep }}=4 M W\right)$, the well is the deepest one and its position appears shifted closer to the separatrix. The velocity shear is stronger at $t=8.6 \mathrm{~s}$ when 
the density gradient is also stronger. Therefore, these density and velocity profiles are 305 consistent with a strong contribution of the $\nabla n / n$ to the formation of the $E_{r}$ profile in 306 this radial area. This tendency is also visible in Figure 9 which is commented in the 307 discussion section. Although it remains rather shallow, the $E \times B$ velocity shear also 308 increases in the inner branch (between $\rho_{\text {pol }}=0.94$ and $\rho_{\text {pol }}=0.985$ ) when the density 309 gradient is the largest. This part of the profile is under investigation, especially to 310 evaluate the contribution of toroidal magnetic ripple (which is about $3 \%$ at the edge in 311 WEST) and its competition with turbulence in generating radial electric field [Var21]. 312

The pedestal in density has been characterized using a fit with a modified tanh 313 function [GO98]. The fit is applied on the inner side of the pedestal only, with no offset ${ }_{314}$ in the edge density. From this fitting procedure, the density at the pedestal and the 315 pedestal width can be determined when reflectometry profiles are available (note that 316 reflectometry profiles measurements are more frequent than DBS). The pedestal width ${ }_{317}$ is defined here as twice the half-width determined from the fit. The temporal evolution 318 of the energy normalized confinement time $H_{98, y 2}$, the power crossing the separatrix, 319 pedestal density, pedestal width and normalized density gradient is shown in Figure 6320 for the pulse 55564 (in LSN) and for the pulse 55562 in USN that is discussed in the ${ }_{321}$ next section. Error bars on $P_{\text {sep }}$ represent the standard deviation over a time window ${ }_{322}$ of $100 \mathrm{~ms}$. We have a reference during the ohmic part of the discharge $(t=3 \mathrm{~s})$, and the ${ }^{323}$ next points are recorded during the high power phase. The transition occurs after the ${ }_{324}$ power crossing the separatrix has reached a value above the expected power threshold for 325 L-H transition [MTI08]. Then the pedestal density increases abruptly and the pedestal ${ }_{326}$ width falls, leading to a sharp increase of the normalized density gradient. Concerning 327 the H-factor $H_{98, y 2}$ which corresponds to the energy confinement time normalised to the ${ }_{328}$ confinement time predicted by the scaling law IPB98 [CCE99] and is valuable only for ${ }_{329}$ the H-mode phases; values are around $H_{98, y 2}=0.6$ for the LSN and $H_{98, y 2}=0.8$ for the ${ }_{330}$ USN case. These low values come from the high level of radiated power which lead to 331 a reduction of the central electron temperature. In order to compensate this effect, the 332 H-factor computed using a net power $P_{t o t}-P_{\text {rad }}$ obtained by subtraction the radiated ${ }_{333}$ power $P_{\text {rad }}$ to the total power $P_{t o t}$ is also given in Figure 6. However, it should be noted ${ }_{334}$ that the use of the $\operatorname{IP98P}(y, 2)$ may be questionable in WEST due to its large aspect ${ }_{335}$ ratio A (around 5-6) and to the relatively narrow range of the available A parameter in ${ }_{336}$ the database.

\subsection{L-H transition observed in USN configuration}

338

Interestingly, a transition is also observed in the so called 'unfavourable $B \times \nabla B$ ', ${ }^{3} 39$ direction therefore in the USN configuration in WEST. This transition appears similar 340 in some points to the L-H transitions occurring in LSN configuration. Notably, an 341 increase of total energy content $W_{M H D}$ (which is weaker than in the LSN transition), a ${ }_{342}$ decrease of the internal inductance, a reduction of the D-gamma signal on the divertor ${ }_{343}$ target as well as a reduction of the density measured by the SOL interferometer chord ${ }_{344}$ 


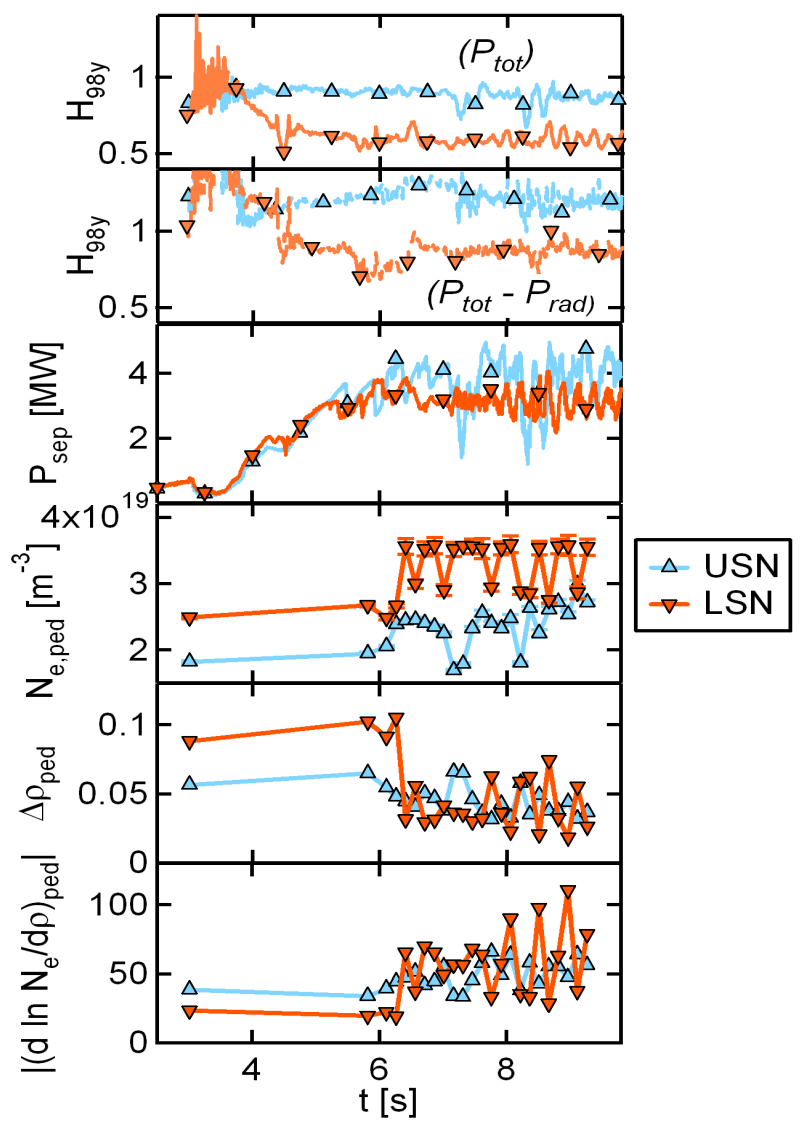

and a deepening of the $E_{r}$ well are simultaneously observed. However, contrary to transitions in LSN discharges, no oscillatory phase takes place during these discharges (see Figure 7). Another difference, which explains the latter, comes from the fact that while the gradient of the density profile clearly increases at the transition, the pedestal in density appears less pronounced than in the LSN case. The reduction in the width of the pedestal density shown in Figure 8 is moderate compared to the LSN case, and the change in the normalized density gradient is also less pronounced (see Figure 6).

Concerning the radial profiles of the $V_{E \times B}$, visible in Figure 8, similarly to the behaviour of the LSN discharge described above, a well is formed in the velocity profile just inside the separatrix at the transition occurring during the ICRH ramp up. The first measurement $(t=6.5 \mathrm{~s})$ after the transition, exhibits a well reaching $-9.5 \mathrm{~km} / \mathrm{s}$. Since the heating power is not stationary $\left(P_{\text {sep }}\right)$ changing between $2 \mathrm{MW}$ and $4.5 \mathrm{MW}$, this discharge is eventful. The 'weakly improved confinement regime' is lost a first time around $t=7.3 \mathrm{~s}$ and this loss starts during the DBS measurements recorded at $t=7.2 \mathrm{~s}$. However, the velocity profile is deeper at this time as compared to the previous time. 

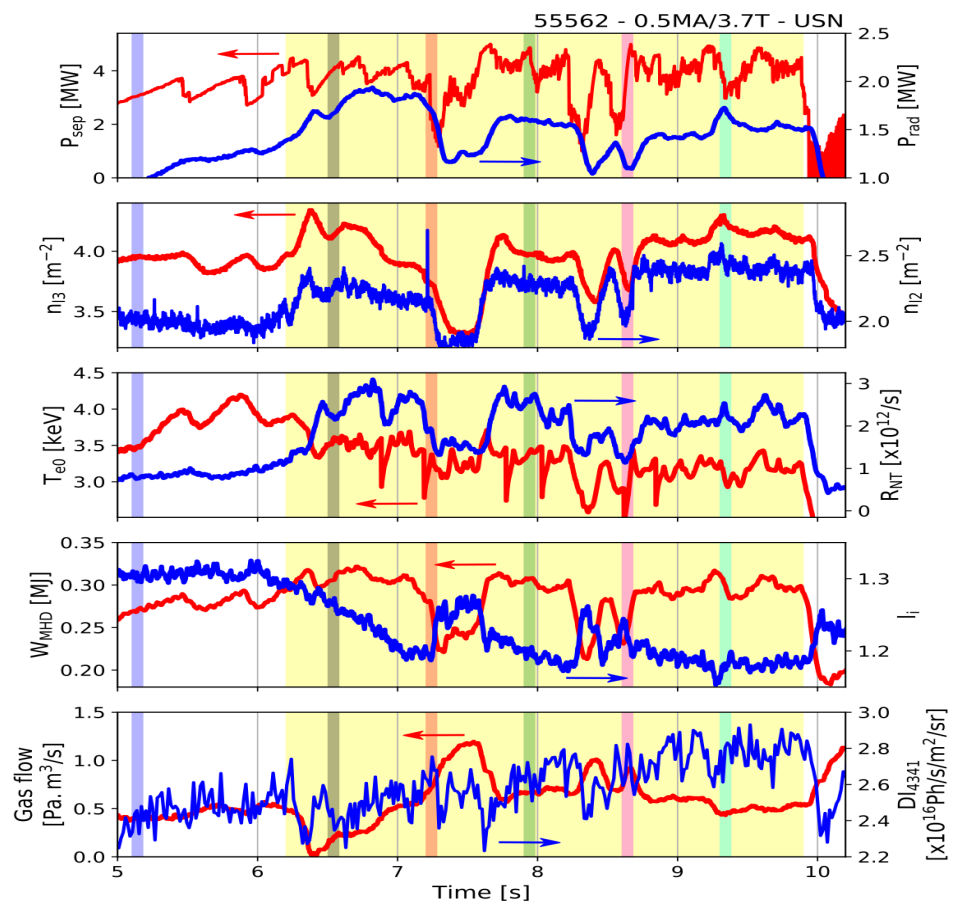

Figure 7. Time traces of radiated power $P_{r a d}$ and power crossing the separatrix $P_{s e p}$, central line integrated densities $n_{l 2}$ and $n_{l 3}$, central electron temperature and neutrons rate $R_{N T}$, total energy content $W_{M H D}$ and internal inductance $l_{i}$, gas flow rate and D-gamma signal on the divertor target $D_{I 4341}$ for a USN discharge with an $\mathrm{L}-\mathrm{H}$ transition at $t=6.2 \mathrm{~s}$. The yellow area shows the $\mathrm{L}-\mathrm{H}$ transition phase and colored vertical lines show the times of the DBS measurements

When enough additional power is recovered $(t=7.55 \mathrm{~s})$, the density increases again and 360 the velocity profile shows anew a well. As compared to the previous one, this velocity 361 well appears weaker while the density profile is similar and $P_{\text {sep }}$ higher. Looking more ${ }_{362}$ in details into the DBS data, it is found that at this time the velocity profile has a ${ }_{363}$ particular behaviour, which is discussed just below. At $t=8.6 \mathrm{~s}$, again the additional 364 power is perturbed and both density profile and velocity profile seem affected by the ${ }_{365}$ change in plasmas condition. After a quieter period in term of heating power, the density ${ }_{366}$ profile exhibits the strongest gradient at the edge of this discharge at $t=9.3 \mathrm{~s}$ and the ${ }_{367}$ velocity profile has a deep well, which remains, however, less pronounced than for the 368 deepest one (corresponding at $t=7.2 s$ ) 369

Therefore, a puzzling result is that during this transition the radial profile of the 370 velocity shows the deepest well observed so far in WEST plasmas. For comparable 371 heating power (mainly $P_{\text {sep }}=3.5 \mathrm{MW}$ ), the $E_{r}$ well is significantly deeper during the ${ }_{372}$ transition in USN than during the one in LSN presented in the previous section, since the ${ }_{373}$ velocity well reaches $-11 \mathrm{~km} / \mathrm{s}$ (against around $-6 \mathrm{~km} / \mathrm{s}$ in LSN). Another interesting 374 aspect is related to the fact that in the same time, the density gradient close to the 375 separatrix is weaker than in the transitions occurring in LSN. 376

Note that the observation of an $E_{r}$ well formation without a clear density pedestal 377 

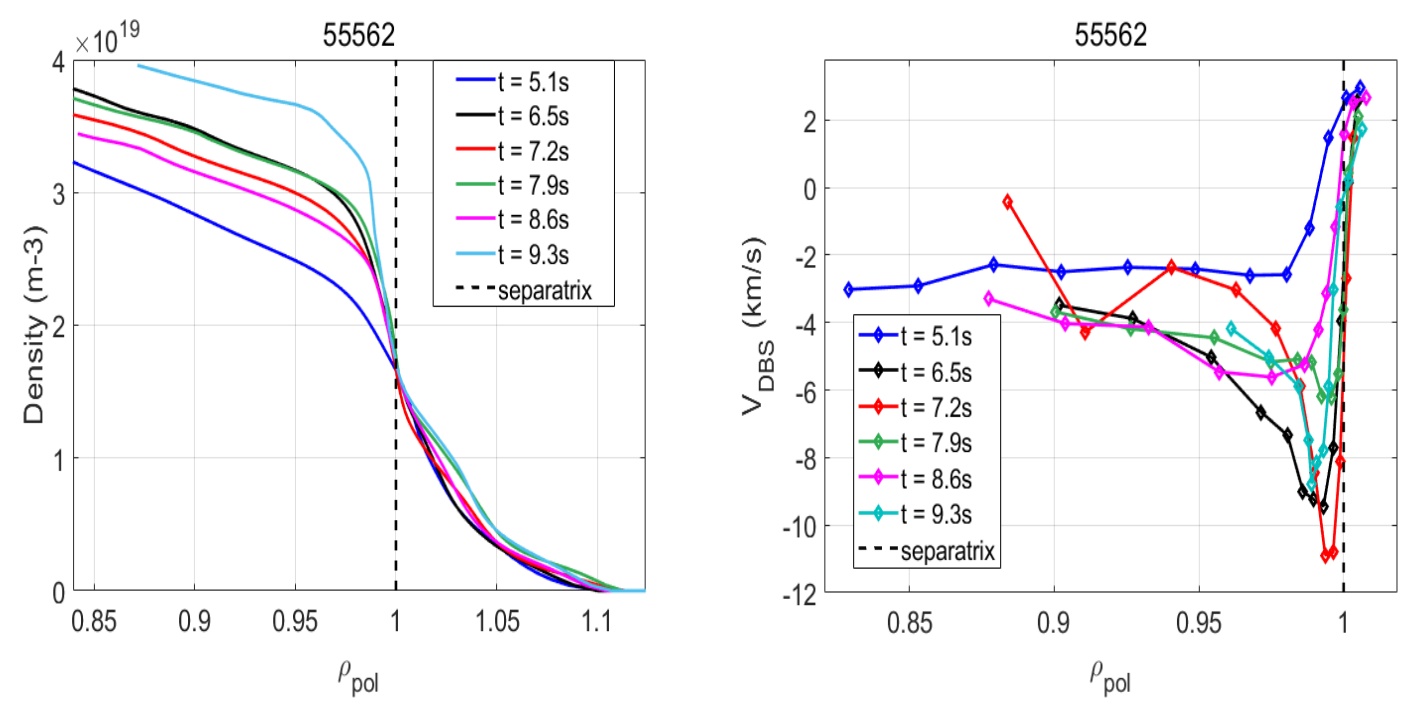

Figure 8. Radial profiles of electron density measured using fast sweep reflectometry $[\mathrm{Cla}+17]$ during an L-H transition phase in USN starting at $t=6.45 \mathrm{~s}$ (left). Associated radial profiles of the $\mathrm{E} \times \mathrm{B}$ velocity measured using DBS [Hen+06] (right). Time measurements are plotted in time traces of the discharge Fig. 7

may suggest that a strong pedestal is formed in the temperature profile. Unfortunately, no measurements of the temperature at the edge are available to verify it. In addition, one could think that this situation suggests an I-mode regime since this configuration is favorable to such mode during which a pedestal forms in temperature only. Especially, WEST operating at high B field, it is expected to have an-I mode significant operational window $[\mathrm{Hub}+16]$. However, in addition to the increase in density, which is not expected during I-mode, no clear signs of a Weakly Coherent Mode typically, considered as the signature of I-mode, is reported on fast sweeping reflectometry.

Concerning the surprising shape of the averaged velocity profile at $t=7.9 \mathrm{~s}$ as compared to the other time measurements, time evolution of the velocity has been analysed more in details. As already mentioned above, the velocity profiles discussed here are averaged profiles, over typically $80 \mathrm{~ms}$ that may not be representative in cases of a specific dynamics. In particular, during this USN discharge (Figure 7), at $t=7.9 \mathrm{~s}$, the DBS amplitude signal exhibits burst events with a frequency around $4 \mathrm{kHz}$, in a radial zone which extends across the well and inner branch. This bursty dynamics is associated with bursts of high (resp. low) Doppler velocities. Therefore, as mentioned just above, the averaged profile (Figure 8, green profile) does not resolve properly the peculiar shape of the velocity profile at this time. This behaviour explains the less deep well. In addition, the dynamics observed both in the velocity and in the amplitude of density fluctuations has strong similitude with observations made in DSB signal on ASDEX Upgrade during I-phase [Med+17]. Note that however, in WEST, no sign of this I-phase dynamics has been detected so far in other signals (such as Dalpha or fast sweep reflectometry). For comparison, during the other measurement times, the velocity 
(at a given probing frequency step, i.e. radial position) also shows a dynamics, which ${ }_{401}$ corresponds more to oscillations around a certain value. This behaviour appears similar 402 to the presence of Geodesic Acoustic Modes (GAMs). The frequency of these oscillations ${ }_{403}$ is around $15 \mathrm{kHz}$ which is higher but of the same order of magnitude of GAMs observed ${ }_{404}$ in Tore Supra [Ver+12]. As no temperature measurements are available, the expected 405 GAM frequency cannot be evaluated for this specific discharge and then does not allow ${ }_{406}$ to confirm or exclude GAMs related oscillations. It should be mentioned that the ${ }_{407}$ amplitude of the DBS appears also modulated suggesting a modulation of the density ${ }_{408}$ in the equatorial plane while this feature is not expected for GAMs (GAMs perturbation ${ }_{409}$ in density is such as $m=1$ ). However, the modulation of DBS amplitude may also result ${ }_{410}$ from an interaction between turbulence at the probing wavenumber and GAMs. ${ }_{411}$

\section{Discussion}

412

In WEST, differences in the shape of the radial profile of $E_{r}$ are observed between LSN and USN configurations. During Ohmic and low heating power discharges, the difference

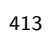
is remarkable ; the $E_{r}$ profile does not exhibit a well in USN while it has a standard shape in LSN. This behaviour changes when increasing the plasma current during Ohmic plasmas or when increasing the addition heating power at fixed plasma current. While the result in Ohmic plasma is consistent with previous observations [Sch+06] and may appear in line with the common belief that configuration with magnetic drift toward $\mathrm{X}$-point is the favourable configuration to access H-mode, it remains unexplained.

First, while no ion temperature measurements are available, the density and electron temperature profiles for these two configurations remain almost identical. Considering the diamagnetic contribution to the radial electric field $\nabla P_{i} / e n_{i}$, taking $T_{i}=T_{e}$, the shape of the $E \times B$ velocity profile should be strongly influenced by the density gradient (in L-mode, $n_{e} \approx n_{i}$ is reasonable). Therefore, at least in one configuration (looking at both density and velocity profiles it is probably in the USN configuration), the observed difference suggests that the $E_{r}$ profile cannot be described using the diamagnetic approximation.

Secondly, when increasing the additional RF heating power, the radial electric field profile becomes more sheared in USN discharges. On this aspect, it should be noted that $\mathrm{RF}$ waves generates a toroidal torque that in turn produces a radial current of particles. This effect was computed in $[\mathrm{Cho}+15]$ and appears quite small in the plasma edge. In 432 the same way, direct losses of energetic particles generated by RF waves are small in ${ }_{433}$ the edge. This reversed situation (deeper well in USN than in LSN) while reaching ${ }^{434}$ plasma conditions for a potential L-H transition is not anymore consistent with the idea ${ }_{435}$ of LSN been a favourable configuration, at least considering that the shear of $E r$ is a ${ }^{436}$ key ingredient of the L-H transition. Indeed this thumb rule would advocate an LSN ${ }_{437}$ configuration as more propicious to a transition, and hence more sheared $E_{r}$ than in ${ }_{438}$ USN. The comparison of the behaviour of $E_{r}$ profile in Ohmic and high power discharges ${ }_{439}$ is not straightforward since conditions are largely different. However, it brings new ${ }_{440}$ 
pieces of understanding in the formation of the $E_{r}$ profile. Especially, the observation of a clear dependence of the velocity shear with the plasma current, stronger in USN than in LSN, is interesting.

The power threshold for the L-H transition does not depend on the plasma current [MTI08], suggesting a weak role of the current in the physics of the $E_{r}$ well formation and sustainment. The sensitivity of the $E_{r}$ profile in Ohmic plasmas with the plasma current may be interpreted as a sign of a substantial change in the physical mechanisms dominating the $E_{r}$ formation. In fact, the plasma current, or more precisely the safety factor, is expected to play some role in the establishment of the radial electric field. Indeed increasing the safety factor (i.e. lowering the current at constant magnetic field) increases the connexion length, and therefore decreases the ability of the plasma to balance charge separation via the particle motion along the field lines (PfirschSchlüter mechanism). Correlatively the orbit width increases with the safety factor, thus presumably leading to a wider $E_{r}$ shear layer if orbit losses (including prompt losses as well as thermal and fast particles losses) are to play a role in the formation of the $E_{r}$ well. This mechanism is also sensitive to the ion $\nabla B$ drift which comes into play when comparing LSN versus LSN configuration. Also the competition between neoclassical viscous damping and turbulent drive plays a role in some models of the L-H transition [Chô+14]. This competition introduces an explicit dependence on the safety factor. In particular, considering the turbulent drive, the breaking of the poloidal symmetry of the magnetic shear with X-point or contact point with a limiter, can induce significant contribution on the $E_{r}$ profile $[\mathrm{Fed}+13$; Man +18$]$. In this spirit, investigation on edge turbulence generating flows via both magnetic and velocity shear, based on a reduced model $[\mathrm{Per}+21]$ are in progress.
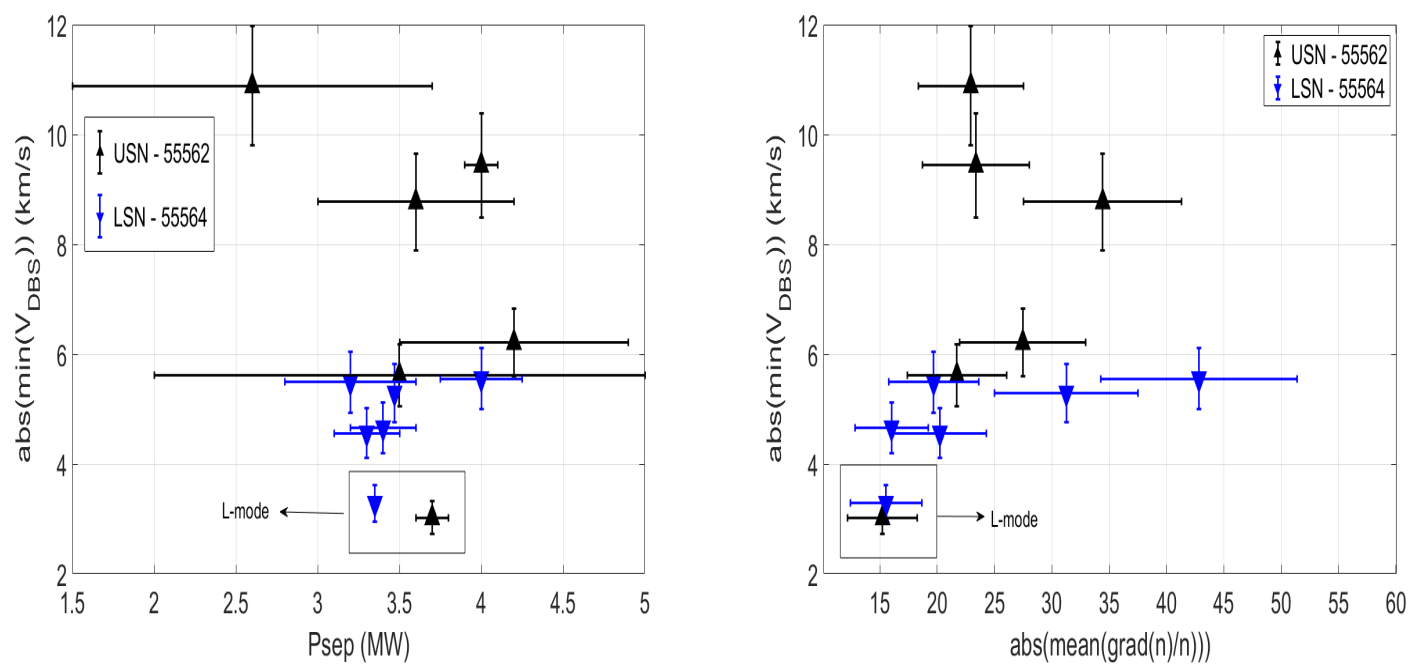

Figure 9. Comparison of the evolution of the depth of the $E_{r}$ well in LSN and USN during $\mathrm{L}$ and $\mathrm{H}$-mode phases as a function of the heating power crossing the separatrix $P_{\text {sep }}$ (left) and as a function of the density gradient at the edge (right) 


\section{References}

[Bur+89] K H Burrell et al. "Confinement physics of H-mode discharges in DIII-D". $\quad{ }^{487}$ In: Plasma Physics and Controlled Fusion 31.10 (Aug. 1989), pp. 1649- ${ }_{488}$ 1664. DOI: 10.1088/0741-3335/31/10/012. URL: https://doi.org/10 . ${ }_{489}$ 1088/0741-3335/31/10/012.

[Wag+82] F. Wagner et al. "Regime of Improved Confinement and High- $\beta$ in Neutral- ${ }_{491}$ Beam-Heated Divertor Discharges of the ASDEX Tokamak". In: Phys. Rev. ${ }_{492}$ Lett. 49 (19 Nov. 1982), pp. 1408-1412. DOI: 10.1103/PhysRevLett.49 . ${ }_{493}$ 1408. URL: https://link.aps.org/doi/10.1103/PhysRevLett.49.1408. 494

[BDT90] H. Biglari, P. H. Diamond, and P. W. Terry. "The Influence of Sheared 495 Poloidal Rotation on Edge Turbulence". In: Phys. Fluids B 2.1 (1990), 496 pp. $1-4$.

[McD +09$]$ R. M. McDermott et al. "Edge radial electric field structure and its 498 connections to H-mode confinement in Alcator C-Mod plasmas". In: Physics 499 of Plasmas 16.5 (2009), p. 056103. DOI: 10 . 1063/1 . 3080721. eprint: 500 
https : //doi.org/10.1063/1.3080721. URL: https ://doi.org/10 . 501 1063/1.3080721.

[Est+09] T Estrada et al. "Sheared flows and transition to improved confinement ${ }_{503}$ regime in the TJ-II stellarator". In: Plasma Physics and Controlled Fusion 51.12 (Nov. 2009), p. 124015. DOI: 10 . 1088/0741-3335/51/12/124015. URL: https://doi .org/10.1088/0741-3335/51/12/124015.

[Vie+13] E. Viezzer et al. "High-accuracy characterization of the edge radial electric field at ASDEX Upgrade". In: Nuclear Fusion 53.5 (2013), p. 053005. URL: http: //stacks. iop.org/0029-5515/53/i=5/a=053005.

$[$ And +08$] \quad Y$. Andrew et al. "Evolution of the radial electric field in a JET H-mode plasma". In: EPL (Europhysics Letters) 83.1 (June 2008), p. 15003. DOI: 10.1209/0295-5075/83/15003. URL: https : //doi .org/10.1209/0295$5075 / 83 / 15003$.

[Kam+11] K. Kamiya et al. "Spatio-temporal structure of the edge radial electric field during H-mode in JT-60U". In: Nuclear Fusion 51.5 (Apr. 2011), p. 053009. DOI: 10 . 1088/0029-5515/51/5/053009. URL: https : //doi . org/10 . 1088/0029-5515/51/5/053009.

[Bur20] K. H. Burrell. "Role of sheared $\mathrm{E} \times \mathrm{B}$ flow in self-organized, improved confinement states in magnetized plasmas". In: Physics of Plasmas 27.6 (2020), p. 060501. DOI: 10.1063/1.5142734. eprint: https ://doi.org/ 10.1063/1.5142734. URL: https://doi.org/10.1063/1.5142734.

$[\mathrm{Cav}+16] \quad$ M. Cavedon et al. "Interplay between turbulence, neoclassical and zonal flows during the transition from low to high confinement mode at ASDEX Upgrade". In: Nuclear Fusion 57.1 (Oct. 2016), p. 014002. DOI: 10.1088/ 0029 - 5515/57 / 1/014002. URL: https : / / doi . org/10 . 1088/0029$5515 / 57 / 1 / 014002$.

$[$ Con +11$]$ G. D. Conway et al. "Mean and Oscillating Plasma Flows and Turbulence Interactions across the L-H Confinement Transition". In: Phys. Rev. Lett. 106 (6 Feb. 2011), p. 065001.

$[$ Sch+12] L. Schmitz et al. "Role of Zonal Flow Predator-Prey Oscillations in Triggering the Transition to H-Mode Confinement". In: Phys. Rev. Lett. 108 (15 Apr. 2012), p. 155002. DOI: 10.1103/PhysRevLett. 108.155002. URL: https://link.aps.org/doi/10.1103/PhysRevLett.108.155002.

[Est +11$] \quad$ T. Estrada et al. "Spatiotemporal Structure of the Interaction between Turbulence and Flows at the L-H Transition in a Toroidal Plasma". In: Phys. Rev. Lett. 107 (24 Dec. 2011), p. 245004. DOI: 10 . 1103 / PhysRevLett .107 .245004. URL: https ://link .aps .org/doi/10 .1103/ PhysRevLett.107.245004.

[KDG91] Y. B. Kim, P. H. Diamond, and R. R. Groebner. "Neoclassical poloidal and toroidal rotation in tokamaks". In: Physics of Fluids B: Plasma Physics 3.8 (1991), p. 2050. 
[SMR11]

[CKW02]

[dMB11]

$[\mathrm{Brz}+19]$

$[$ Gar+10]

$[\mathrm{Ura}+11]$

$[$ Fen +11$]$

$[\mathrm{Nav}+10]$ https://doi.org/10.1063/1.3454365. flows in toroidal plasmas". In: Plasma Physics and Controlled Fusion 53.2 (Jan. 2011), p. 024006. DOI: 10 . 1088/0741-3335/53/2/024006. URL: https://doi .org/10.1088/0741-3335/53/2/024006.

C. S. Chang, Seunghoe Kue, and H. Weitzner. "X-transport: A baseline nonambipolar transport in a diverted tokamak plasma edge". In: Physics of Plasmas 9.9 (2002), pp. 3884-3892. DOI: 10.1063/1.1490348. eprint: https : //doi .org/10 .1063/1.1490348. URL: https ://doi .org/10 . 1063/1.1490348.

J.S. deGrassie, S.H. Müller, and J.A. Boedo. "Plasma flow due to a losscone distribution centred around the outboard edge in DIII-D". In: Nuclear Fusion 52.1 (Dec. 2011), p. 013010. DOI: 10.1088/0029-5515/52/1/ 013010. URL: https://doi.org/10.1088/0029-5515/52/1/013010.

Robert W. Brzozowski et al. "A geometric model of ion orbit loss under the influence of a radial electric field". In: Physics of Plasmas 26.4 (2019), p. 042511. DOI: 10.1063/1.5075613. eprint: https://doi.org/10.1063/ 1.5075613. URL: https://doi.org/10.1063/1.5075613.

$\mathrm{X}$. Garbet et al. "Entropy production rate in tokamaks with nonaxisymmetric magnetic fields". In: Physics of Plasmas 17.7 (2010), p. 072505. DOI: 10.1063/1.3454365. eprint: https://doi.org/10.1063/1.3454365. URL:

M. F. F. Nave et al. "Influence of Magnetic Field Ripple on the Intrinsic
Rotation of Tokamak Plasmas". In: Phys. Rev. Lett. 105 (10 Sept. 2010), p. 105005. DOI: 10.1103/PhysRevLett. 105.105005. URL: https://link. aps.org/doi/10.1103/PhysRevLett.105.105005.

H. Urano et al. "Edge pedestal characteristics in JET and JT-60U tokamaks 567 under variable toroidal field ripple". In: Nuclear Fusion 51.11 (Oct. 2011), ${ }_{568}$ p. 113004. DOI: $10.1088 / 0029-5515 / 51 / 11 / 113004$. URL: https://doi. 569 org/10.1088/0029-5515/51/11/113004.

570

C. Fenzi et al. "On plasma rotation with toroidal magnetic field ripple ${ }_{571}$ and no external momentum input". In: Nuclear Fusion 51.10 (Sept. 2011), 572 p. 103038. DOI: $10.1088 / 0029-5515 / 51 / 10 / 103038$. URL: https://doi . 573 org/10.1088/0029-5515/51/10/103038. 574

[Mon+97] P Monier-Garbet et al. "Effects of neutrals on plasma rotation in DIII-D". 575 In: Nuclear Fusion 37.3 (Mar. 1997), pp. 403-412. DOI: 10.1088/0029- 576 5515/37/3/i09. URL: https://doi.org/10.1088/0029-5515/37/3/i09. 577

[DK91] P. H. Diamond and Y.-B. Kim. "Theory of mean poloidal flow generation 578 by turbulence". In: Phys. Fluids B 3.7 (1991), pp. 1626-1633.
544

545

546 547 548 549 551 :

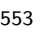

554 560 57

558

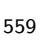

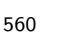
an

562

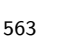
.

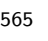

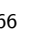
, (1) 
[Dif+09] G. Dif-Pradalier et al. "Interplay between Gyrokinetic Turbulence, Flows, 580 and Collisions: Perspectives on Transport and Poloidal Rotation". In: Phys. Rev. Lett. 103 (6 Aug. 2009), p. 065002. DOI: 10.1103/PhysRevLett. 103. 065002. URL: https://link.aps.org/doi/10.1103/PhysRevLett. 103. 065002.

[Gri+13] B.A. Grierson et al. "Collisionality scaling of main-ion toroidal and poloidal rotation in low torque DIII-D plasmas". In: Nuclear Fusion 53.6 (May 2013), p. 063010. DOI: 10 1088/0029-5515/53/6/063010. URL: https : //doi.org/10.1088/0029-5515/53/6/063010.

$[$ Fed +12$] \quad$ N. Fedorczak et al. "Shear-induced Reynolds stress at the edge of L-mode tokamak plasmas". In: Nuclear Fusion 52.10 (Sept. 2012), p. 103013. DOI: 10.1088/0029-5515/52/10/103013. URL: https://doi.org/10.1088/ 0029-5515/52/10/103013.

[Cha+17] A V Chankin et al. "EDGE2D-EIRENE modelling of near SOL E r: possible impact on the H-mode power threshold". In: Plasma Physics and Controlled Fusion 59.4 (Mar. 2017), p. 045012. DOI: 10.1088/1361-6587/aa5ecc. URL: https://doi.org/10.1088/1361-6587/aa5ecc.

$[$ Buc +14$]$ J. Bucalossi et al. "The WEST project: Testing ITER divertor high heat flux component technology in a steady state tokamak environment". In: Fusion Engineering and Design 89.7 (2014). Proceedings of the 11th International Symposium on Fusion Nuclear Technology-11 (ISFNT-11) Barcelona, Spain, 15-20 September, 2013, pp. 907-912. ISSN: 0920-3796. DOI: https : / / doi . org / 10 . 1016/j . fusengdes . 2014 . 01.062. URL: https : / / www . sciencedirect . com / science / article / pii / S0920379614000635.

[Tri+08] E. Trier et al. "Radial electric field measurement in a tokamak with magnetic field ripple". In: Nuclear Fusion 48.9 (Aug. 2008), p. 092001. DOI: 10 . 1088/0029-5515/48/9/092001. URL: https : //doi .org/10 . 1088/0029-5515/48/9/092001.

[Ver +18$] \quad$ L. Vermare et al. "Poloidal asymmetries of flows in the Tore Supra tokamak". In: Physics of Plasmas 25.2 (2018), p. 020704. DOI: 10.1063/ 1.5022122. eprint: https://doi.org/10.1063/1.5022122. URL: https: //doi.org/10.1063/1.5022122.

[Hen+10] P. Hennequin et al. "The effect of SOL flows on edge and core radial electric field and rotation in Tore Supra". In: 37th EPS Conference on Plasma Physics, Dublin 34A, P1.1040 (2010).

[Hen+06] P. Hennequin et al. "Fluctuation spectra and velocity profile from Doppler backscattering on Tore Supra". In: Nuclear Fusion 46.9 (2006), S771.

[Hon+06] C. Honoré et al. "Quasi-optical Gaussian beam tracing to evaluate Doppler backscattering conditions". In: Nuclear Fusion 46.9 (2006), S809-S815. 
${ }_{620}[\mathrm{Cla}+17]$

[Fau20]

$[\operatorname{Sch}+06]$

$[$ Fed +13$]$

[MTI08]

[CCE99]

$[$ Ryt +13$]$

[Vin19]

[Put20]

$[\mathrm{Cla}+01]$
F. Clairet et al. "1 $\mu$ s broadband frequency sweeping reflectometry for plasma density and fluctuation profile measurements". In: Review of ${ }_{621}$ Scientific Instruments 88.11 (2017), p. 113506. DOI: 10.1063/1.4991789. ${ }_{622}$ eprint: https://doi.org/10.1063/1.4991789. URL: https://doi.org/ ${ }_{623}$ 10.1063/1.4991789.

624

Blaise Faugeras. "An overview of the numerical methods for tokamak ${ }_{625}$ plasma equilibrium computation implemented in the NICE code". In: ${ }_{626}$ Fusion Engineering and Design 160 (2020), p. 112020. ISSN: 0920-3796. DOI: ${ }_{627}$ https://doi.org/10.1016/j.fusengdes.2020.112020. URL: https: ${ }_{628}$ //www.sciencedirect.com/science/article/pii/S0920379620305688. ${ }_{629}$

J Schirmer et al. "The radial electric field and its associated shear in the 630 ASDEX Upgrade tokamak". In: Nuclear Fusion 46.9 (Aug. 2006), S780- ${ }_{631}$ S791. DOI: $10.1088 / 0029-5515 / 46 / 9 /$ s13. URL: https://doi.org/10. 632 1088/0029-5515/46/9/s13.

633

$\mathrm{N}$ Fedorczak et al. "Dynamics of tilted eddies in a transversal flow at the ${ }_{634}$ edge of tokamak plasmas and the consequences for L-H transition". In: ${ }_{635}$ Plasma Physics and Controlled Fusion 55.12 (Nov. 2013), p. 124024. DOI: 636 10.1088/0741-3335/55/12/124024. URL: https://doi.org/10.1088/ 637 0741-3335/55/12/124024.

638

Y R Martin, T Takizuka, and the ITPA CDBM H-mode Threshold Data ${ }_{639}$ Group. "Power requirement for accessing the H-mode in ITER". In: Journal ${ }_{640}$ of Physics: Conference Series 123 (July 2008), p. 012033. DOI: 10.1088/ ${ }_{641}$ 1742-6596/123/1/012033. URL: https : / doi .org/10 . 1088/1742- 642 6596/123/1/012033.

643

ITER Physics Expert Group on Confin Transport, ITER Physics Expert ${ }_{644}$ Group on Confin Database, and ITER Physics Basis Editors. "Chapter 2: ${ }_{645}$ Plasma confinement and transport". In: Nuclear Fusion 39.12 (Dec. 1999), 646 pp. 2175-2249. DOI: $10.1088 / 0029-5515 / 39 / 12 / 302$. URL: https://doi. ${ }_{647}$ org/10.1088/0029-5515/39/12/302. ${ }_{648}$ F. Ryter et al. "Survey of the H-mode power threshold and transition ${ }_{649}$ physics studies in ASDEX Upgrade". In: Nuclear Fusion 53.11 (Sept. 2013), 650 p. 113003. DOI: 10.1088/0029-5515/53/11/113003. URL: https://doi. ${ }_{651}$ org/10.1088/0029-5515/53/11/113003.

T. Putterich. In: ITPA Transport and confinement, June 2020 (2020). ${ }_{654}$ $\mathrm{F}$ Clairet et al. "Edge density profile measurements by X-mode 655 reflectometry on Tore Supra". In: Plasma Physics and Controlled Fusion 656 43.4 (Mar. 2001), pp. 429-441. DOI: 10.1088/0741-3335/43/4/305. URL: ${ }_{657}$ https://doi.org/10.1088/0741-3335/43/4/305. 658

[Var21] R. Varennes. In: Private Communication (2021). 659 
[GO98] R. J. Groebner and T. H. Osborne. "Scaling studies of the high mode 660 pedestal". In: Physics of Plasmas 5.5 (1998), pp. 1800-1806. DOI: 10 . 1063/1 . 872849. eprint: https : / / doi . org/10 . 1063/1 . 872849. URL: https://doi.org/10.1063/1.872849.

$[\mathrm{Hub+16}]$ A.E. Hubbard et al. "Multi-device studies of pedestal physics and confinement in the I-mode regime". In: Nuclear Fusion 56.8 (July 2016), p. 086003. DOI: 10.1088/0029-5515/56/8/086003. URL: https://doi . org/10.1088/0029-5515/56/8/086003.

$[$ Med+17] A Medvedeva et al. "Density profile and turbulence evolution during L$\mathrm{H}$ transition studied with the ultra-fast swept reflectometer on ASDEX Upgrade". In: Plasma Physics and Controlled Fusion 59.12 (Nov. 2017), p. 125014. DOI: 10.1088/1361-6587/aa9251. URL: https://doi .org/10. 1088/1361-6587/aa9251.

[Ver+12] L Vermare et al. "Detection of Geodesic Acoustic Mode oscillations, using MUltiple Signal Classification analysis of Doppler backscattering signal on Tore Supra". In: Nuclear Fusion 52.6 (2012), p. 063008.

[Cho+15] B Chouli et al. "Investigations of LHCD induced plasma rotation in Tore Supra". In: 57.12 (Oct. 2015), p. 125007. DOI: 10.1088/0741-3335/57/ 12/125007. URL: https://doi.org/10.1088/0741-3335/57/12/125007.

[Chô+14] L. Chôné et al. "L-H transition dynamics in fluid turbulence simulations with neoclassical force balance". In: Physics of Plasmas 21.7 (2014), p. 070702. DOI: 10 .1063/1.4890971. eprint: https : / / doi . org/10 . 1063/1.4890971. URL: https://doi.org/10.1063/1.4890971.

[Man+18] P. Manz et al. "Magnetic configuration effects on the Reynolds stress in the plasma edge". In: Physics of Plasmas 25.7 (2018), p. 072508. Dor: 10.1063/1.5037511. eprint: https://doi .org/10.1063/1.5037511. URL: https://doi.org/10.1063/1.5037511.

$[$ Per +21$] \quad$ M. Peret et al. "A spectral model for interchange transport in tokamak scrape-off layers". In: Nuclear Fusion 61.4 (Mar. 2021), p. 046045. DOI: 10. 1088/1741-4326/abe6b3. URL: https : / / doi .org/10 . 1088/17414326/abe6b3. 